\title{
lon-induced formation of regular nanostructures on amorphous GaSb surfaces
}

\author{
S. Facsko, a) T. Bobek, and H. Kurz \\ Lehrstuhl und Institut für Halbleitertechnik, RWTH Aachen, Sommerfeldstr. 24, 52056 Aachen, Germany \\ T. Dekorsy \\ Institut für Ionenstrahlphysik und Materialforschung, Forschungszentrum Rossendorf, Postfach 510119, \\ 01314 Dresden, Germany
}

\author{
S. Kyrsta and R. Cremer \\ Institut für Theoretische Hüttenkunde, RWTH Aachen, 52056 Aachen, Germany
}

\begin{abstract}
Crystalline and amorphous GaSb surfaces are compared concerning their response to sputter erosion with low energy $\mathrm{Ar}^{+}$ions under normal incidence. We show that the formation of regular nanostructures on $\mathrm{GaSb}$ is basically independent of whether the initial material is crystalline or amorphous. The similarity in the temporal and spatial evolution demonstrates that the dynamics of the morphology evolution is entirely controlled by a thin amorphous surface layer.
\end{abstract}

The large potential of nanoelectronics has stimulated much effort to find new methods for the parallel processing of nanostructures. Promising techniques, like StranskiKrastanov growth of semiconductor heterostructures ${ }^{1}$ and self-assembly of semiconductor nanocrystals by colloid chemistry $^{2}$ have been exploited. Recently, during the erosion of semiconductor and metal surfaces by ion sputtering, a self-organizing mechanism has been discovered leading to regular patterns of structures with dimensions of some tens of nanometers. ${ }^{3,4}$ These surface structures can form quantum dots with a high aspect ratio and are therefore particularly attractive for quantum electronic applications.

The appearance of periodic ripple patterns on semiconductor and metal surfaces bombarded with ions under offnormal incidence has been known for a long time. ${ }^{5}$ In certain metals and III-V semiconductors these ripple patterns transform into hexagonally ordered isolated nanostructures under normal incidence sputtering. ${ }^{3,4,6-8} \mathrm{Up}$ to now these observations have only been made in crystalline materials. The main points addressed in this letter concern (i) the comparison of the pattern evolution from initially crystalline $(c-\mathrm{GaSb})$ and amorphous $\mathrm{GaSb}(\alpha-\mathrm{GaSb})$ targets and (ii) the microscopic structure of the patterned surface. We find that the surface dot patterns generated are independent of the crystallinity of the initial material surface. This independence is explained by the decisive role of a thin amorphous layer that forms during the very first seconds of the sputtering process. Besides the fundamental insight into the sputtering process on semiconductor surfaces, the structuring of amorphous layers deposited on arbitrary materials provides a technologically attractive method of surface nanostructuring.

Stochastic nonlinear continuum models have been introduced to explain the temporal and spatial evolution of nanoscale surface patterns during ion sputtering ${ }^{9}$

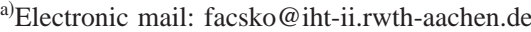

$$
\begin{aligned}
\frac{\partial h}{\partial t}= & -v_{0}+\nu \nabla^{2} h+\frac{\lambda(\theta)}{2}(\nabla h)^{2}+D \nabla^{2}\left(\nabla^{2} h\right) \\
& +\eta(x, y, t),
\end{aligned}
$$

where $h(x, y)$ is the surface height function, $v_{0}$ is the constant erosion velocity, $\nu$ is the "negative surface tension," $\lambda(\theta)$ is a nonlinear coefficient attributed to the tilt dependent sputter yield with $\theta$ the tilt angle, $D$ is the thermal or ion induced surface diffusion, and $\eta$ denotes a noise term, that describes the stochastic character of the sputtering process. This continuum equation represents an approximation to the surface dynamics because higher order derivatives are not considered. The effect of additional higher order terms in Eq. (1) is believed to be minor. ${ }^{10}$ The microscopic model adopted for the interpretation of the coefficients in Eq. (1) is the surface instability caused by the inhomogeneous deposition of the ion energy into the surface. ${ }^{9,11}$ In this picture the coefficients of Eq. (1), $v_{0}, \nu, D$, and $\lambda$ are determined by ensemble averages of the microscopic parameters of the distribution of the energy deposited by the ions. The negative surface tension $\nu$ originates from the curvature dependence of the sputtering yield, which is proportional to the total energy deposited and therefore higher in the valleys than on the hills. A stability analysis reveals that a narrow wavelength range centered at $l_{c}=2 \pi \sqrt{2 D /|\nu|}$ is amplified out of the spectrum of the initial surface roughness, driving the surface to a rippled morphology in the anisotropic case (oblique incidence) and to a hexagonal arrangement of regular dots in the isotropic case (normal incidence or rotation of the sample under oblique incidence), ${ }^{3,8,9}$

The experimental setup used in the ion sputtering experiments consists of a sputtered neutral mass spectrometer. The $\mathrm{Ar}^{+}$ions used for the sputtering are generated in an electron cyclotron resonance plasma, and are extracted directly from the plasma to the sample surface at normal incidence. The ion energy is varied from 100 to $1800 \mathrm{eV}$, with a typical ion flux of $1 \times 10^{16} \mathrm{~cm}^{-2} \mathrm{~s}^{-1}$. During the continuous sputtering 

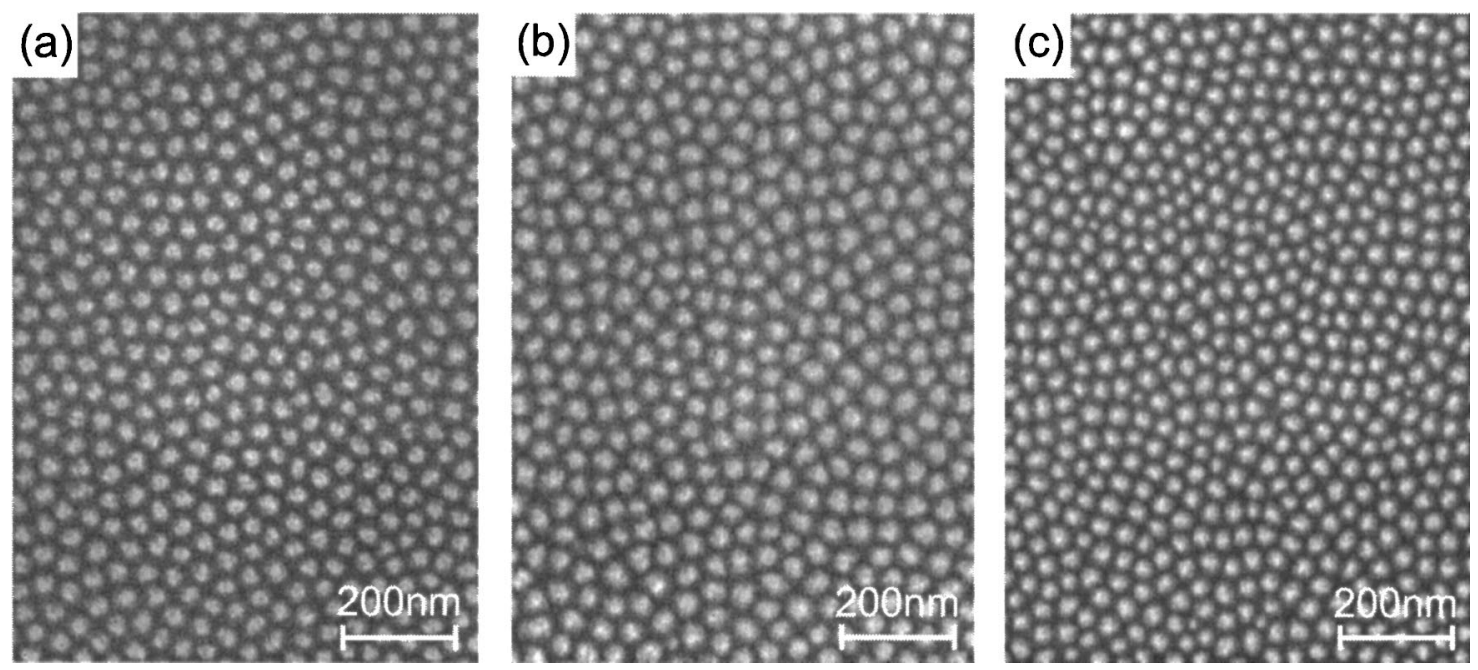

FIG. 1. Scanning electron micrographs of dot patterns on GaSb films: 1 (a) the initial layer is $c$-GaSb(100), 1(b) the initial layer is $c$-GaSb(111), $1(\mathrm{c})$ the initial layer is amorphous $\mathrm{GaSb}$ deposited on $\mathrm{Si}(111)$. The patterns are created at an $\mathrm{Ar}^{+}$-ion energy of $500 \mathrm{eV}$, an ion flux of $1 \times 10^{16} \mathrm{~cm}^{-2} \mathrm{~s}^{-1}$, and an erosion time of $200 \mathrm{~s}$.

the sample mounting is cooled by water, keeping the sample temperature below $100^{\circ} \mathrm{C}$.

The $c$-GaSb samples, that were used in the sputtering experiments, are commercial GaSb wafers with (100) and (111) orientation. Prior to sputtering they are rinsed in ethanol. Additionally, stoichiometric amorphous GaSb films were deposited from a $\mathrm{GaSb}$ target on $\mathrm{Si}(111)$ wafers in a magnetron sputtering chamber at a substrate temperature of $25^{\circ} \mathrm{C}$. The thickness of the $\alpha$-GaSb films was $560 \mathrm{~nm}$. The base pressure of the sputtering system was $<1 \times 10^{-5} \mathrm{~Pa}$ and the distance between substrate and target $8 \mathrm{~cm}$. Argon was used for sputtering the GaSb target (purity 99.9\%) in dc mode. The resulting growth rate at an applied power of $14 \mathrm{~W}$ was $0.2 \mathrm{~nm} \mathrm{~s}^{-1}$. The amorphous structure of the deposited films was verified by coherent phonon spectroscopy. ${ }^{12}$

The sputter process relevant to the formation of dot patterns on $\mathrm{GaSb}$ surfaces is confined to the subsurface volume, where the ions deposit their energy. In the low energy range considered here $(100-1800 \mathrm{eV})$, this interaction layer is between 1 and $4 \mathrm{~nm}$ in thickness as predicted by Monte Carlo simulations with transport of ions in matter (TRIM). ${ }^{13}$ The formation from initially crystalline surfaces starts with significant changes in the surface stoichiometry due to preferential sputtering, followed by a rapid amorphization driven by defect generation. At an ion flux density of $10^{16} \mathrm{~cm}^{-2} \mathrm{~s}^{-1}$ these two steps occur in the first second of the sputtering process. The amorphization of semiconductors reaches a saturation at an ion dose of $1-5 \times 10^{15} \mathrm{~cm}^{-2}$ (estimated from experimental data on $\mathrm{Si}$ and GaAs surfaces ${ }^{14}$ and defect generation rates by $\operatorname{TRIM}^{13}$ ). In our experiments this translates to an amorphization time smaller than $1 \mathrm{~s}$. The saturation fluence for compositional changes due to pure preferential sputtering is of the order of $1 \times 10^{15} \mathrm{~cm}^{-2}$, too, i.e., the removal of $1 \mathrm{ML}$ by the sputtering process. ${ }^{15}$ During erosion there is a continuous transfer from the $c$ state to the $\alpha$ state whose reaction time constant is by far less than that of the erosion. As the erosion proceeds with a rate of $1.8 \mathrm{~nm} / \mathrm{s}$ the hexagonally ordered dot pattern is formed within $20 \mathrm{~s}$ at an ion energy of $500 \mathrm{eV}$, evolving into a steady-state topography after approximately $200 \mathrm{~s}^{7}$ In Fig. 1(a) a scanning elec- tron microscope (SEM) image of the pattern developed in the case of $c-\mathrm{GaSb}(100)$ is shown as a typical example for a $200 \mathrm{~s}$ exposure at $500 \mathrm{eV}$ ion energy. The patterns are covered by an amorphous GaSb layer, whose thickness of $2 \mathrm{~nm}$ has been determined by cross-sectional transmission electron microscopy. ${ }^{3}$ Auger electron spectroscopy of the sputtered surfaces reveals a $\mathrm{Ga}$ enrichment in the first $2-3 \mathrm{~nm}$. The height and diameter of the dot structures depend principally on ion energy and are $15-80 \mathrm{~nm}$ in the energy range considered here. ${ }^{7}$

We propose that the pattern formation and evolution depend entirely on the properties of the thin amorphous layer formed during sputtering. Hence the coefficients in Eq. (1), $\nu, D$, and $\lambda(\theta)$ should reflect the microscopic nature of the sputtering process in the amorphous layer solely. Thus, contrary to the case of metals, the symmetry of the dot pattern in $\mathrm{GaSb}$ and other III-V compounds should appear completely decoupled from the symmetry of the crystalline surface. ${ }^{16}$ The validity of this picture is confirmed by the comparison of dot patterns formed on (100) and (111) GaSb surfaces, which exhibit identical patterns after $200 \mathrm{~s}$ sputtering with $500 \mathrm{eV} \mathrm{Ar}^{+}$ions, as shown in Figs. 1(a) and 1(b).

This result is further corroborated by ion-induced formation of hexagonal patterns on initially amorphous films deposited on arbitrary substrates. We investigated $\alpha$-GaSb films on $\mathrm{Si}(111)$ with a thickness of $560 \mathrm{~nm}$. In Fig. 1(c) the pattern formed on $\alpha$-GaSb under identical experimental conditions is shown for comparison with the pattern formed on $c$-GaSb. The sputtering time for removal of the $\alpha$-GaSb film at these conditions is $310 \mathrm{~s}$. There is a close resemblance between the SEM pictures of the dot patterns. The striking similarity proves conclusively that the pattern formation is defined by a thin amorphous GaSb surface layer, which in the case of $\alpha-\mathrm{GaSb}$ is amorphous from the beginning, whereas in the case of $c$ - $\mathrm{GaSb}(100)$ and (111) is formed before the pattern formation starts. Similar results are obtained at other energies in the range of 100 to $1800 \mathrm{eV}$ with the sputtering times adjusted to give the same thickness of eroded material.

In conclusion, we observed the independence of the pat- 
tern formation process on crystalline and amorphous GaSb. We conclude that the formation of the amorphous layer governs the surface dynamics for both cases. The possibility to form highly dense nanostructures by ion sputtering of amorphous semiconductor films offers attractive potential for a simple self-masking nanotechnology. In combination with appropriate and sufficiently selective etching processes these nanodot patterns can be transferred in arbitrary materials expending the range of possible applications considerably.

The authors would like to thank C. Zanke for the SEM analysis and M. Först for careful analysis of coherent phonon spectra. This work was supported by the Deutsche Forschungsgemeinschaft under the Contract No. KU 540/41-1. T.D. acknowledges support through the Bennigsen-Foerder award of the state North-Rhine Westphalia.

${ }^{1}$ V. A. Shchukin and D. Bimberg, Rev. Mod. Phys. 71, 1125 (1999).

${ }^{2}$ P. Alivisatos, Pure Appl. Chem. 72, 3 (2000).

${ }^{3}$ S. Fascko, T. Dekorsy, C. Koerdt, C. Trappe, H. Kurz, A. Vogt, and H. Hartnagel, Science 285, 1551 (1999).

${ }^{4}$ S. Rusponi, G. Costantini, F. B. de Mongeot, C. Boragno, and U. Valbusa, Appl. Phys. Lett. 75, 3318 (1999).

${ }^{5}$ M. Navez, C. Sella, and D. Chaperot, C. R. Acad. Sci. Paris 254, 240 (1962); F. Vasiliu, I. A. Teodoescu, and F. Glodeanu, J. Mater. Sci. 8, 1030 (1975); G. Carter and V. Vishnyakov, Phys. Rev. B 54, 17647 (1996). E.
Chason, T. M. Mayer, B. K. Kellerman, D. T. McIlroy, and A. J. Howard, Phys. Rev. Lett. 72, 3040 (1994).

${ }^{6}$ M. V. R. Murty, T. Curcic, A. Judy, B. H. Cooper, A. R. Woll, J. D. Brock, S. Kycia, and R. L. Headrick, Phys. Rev. Lett. 80, 4713 (1998).

${ }^{7}$ S. Facsko, H. Kurz, and T. Dekorsy, Phys. Rev. B 63, 165329 (2001).

${ }^{8}$ F. Frost, A. Schindler, and F. Bigl, Phys. Rev. Lett. 85, 4116 (2000).

${ }^{9}$ R. M. Bradley and J. M. E. Harper, J. Vac. Sci. Technol. A 6, 2390 (1988); M. A. Makeev and A.-L. Barabási, Appl. Phys. Lett. 71, 2800 (1997); B. Kahng, H. Jeong, and A.-L. Barabási, ibid. 78, 805 (2000); M. Makeev, R. Cuerno, and A.-L. Barabási, Morphology of Ion-Sputtered Surfaces, http:// xxx.lanl.gov/abs/cond-mat/0007354, 2000.

${ }^{10}$ G. Carter, Phys. Rev. B 59, 1669 (1998).

${ }^{11}$ P. Sigmund, J. Mater. Sci. 8, 1545 (1973).

${ }^{12}$ The phonon spectra of the $\alpha$-GaSb were compared to phonon spectra of $c$-GaSb wafers. The deposited $\alpha$-GaSb films show a broad phonon spectrum characteristic for a noncrystalline solid in contrast to the sharp TOand LO-phonon frequencies at 6.8 and $7.0 \mathrm{THz}$ for $c$-GaSb. For details of the method see M. Först, T. Dekorsy, C. Trappe, M. Laurenzis, H. Kurz, and M. Bechevet, Appl. Phys. Lett. 77, 1964 (2000).

${ }^{13}$ J. F. Ziegler, J. P. Biersack, and U. Littmark, The Stopping and Range of Ions in Matter (Pergamon, New York, 1985).

${ }^{14} \mathrm{H}$. Gnaser, Low-Energy Ion Irradiation of Solid Surfaces, Vol. 146, Springer Tracts in Modern Physics (Springer, Berlin, 1999).

${ }^{15}$ A. Benninghoven, F. G. Rüdenauer, and H. W. Werner, Secondary Ion Mass Spectrometry (Wiley, New York, 1987).

${ }^{16}$ Metal surfaces remain almost crystalline during sputtering at room temperature. For the pattern formation it is concluded therefore that ioninduced patterns on metals will reflect the symmetry of the crystal (Ref. 4). 